\title{
The Effects of Moufarrege Total Posterior Pedicle Reduction Mammaplasty on Breast Cancer Incidence
}

\author{
Charles Maalouf, Richard Moufarrege \\ Department of Plastic Surgery, Montreal University Hospital Center (CHUM), Montreal, Canada \\ Email: Plasticsurg_moufar@hotmail.com
}

How to cite this paper: Maalouf, C. and Moufarrege, R. (2017) The Effects of Moufarrege Total Posterior Pedicle Reduction Mammaplasty on Breast Cancer Incidence. Surgical Science, 8, 133-144. https://doi.org/10.4236/ss.2017.83015

Received: January 13, 2017

Accepted: March 3, 2017

Published: March 6, 2017

Copyright $\odot 2017$ by authors and Scientific Research Publishing Inc. This work is licensed under the Creative Commons Attribution International License (CC BY 4.0).

http://creativecommons.org/licenses/by/4.0/

(c) (i) Open Access

\begin{abstract}
Introduction: The Moufarrege total posterior pedicle breast reduction involves complete denudation of the breast offering free access to all breast quadrants. This allows a total resection of the upper outer quadrant which is most implicated in breast cancer. The objective was to assess the postoperative incidence of breast cancer in the cohort of women who underwent the Moufarrege technique. Methods: Retrospective chart review was performed for patients operated on between 1981 and 1997, and a written questionnaire was sent to all patients in 2008. Patients were asked about the date of their last mammogram and any diagnosis of breast cancer. Expected numbers of cancers were calculated from rates in the general population. Age standardization at the time of surgery was done and the incidence of breast cancer was calculated in relation to age at time of surgery and age at last follow-up. Results were presented as standardized incidence ratios (SIR) with their corresponding 95\% confidence interval (CI). Results: We included 924 patients. The response rate was $60.38 \%(n=558)$. Mean patient age at the time of the surgery was 32.8 years (14 to 82). Mean resection weight was 620 grs per breast (250 $1600)$. Breast cancer was significantly reduced by nearly 64 percent in patients who responded to our questionnaire and were aged 35 years or more at the time of surgery $([\mathrm{SIR}]=0.36 ; 95 \%[\mathrm{CI}] 0.09-0.93)$. No difference in breast cancer incidence was observed in patients aged less than 25 years at the time of the surgery. Conclusion: The Moufarrege Total Posterior Pedicle breast reduction is a reliable and safe procedure that may offer a superior breast cancer risk reduction based on the results of our retrospective chart review and patient questionnaire.
\end{abstract}

\section{Keywords}

Moufarrege Total Posterior Pedicle, Breast Reduction, Reduction Mammoplasty, Breast Cancer 


\section{Introduction}

Breast reduction (BR) or reduction mammaplasty is a surgical procedure involving the excision of excess breast parenchyma, fatty tissue and skin in the aim of enhancing functional, aesthetic, as well as psycho-social outcome. Besides its aesthetic role in patients with gigantomastia or breast hypertrophy, breast reduction was shown to effectively relieve symptoms such as back or shoulder pain, and offer an overall improvement in the quality of life [1] [2] [3]. BR is also often performed for symmetrisation of the contralateral breast in patients who had breast reconstruction following mastectomy for cancer. In this group of patients and all patients with increased risk for breast cancer, the reduction in risk of subsequent breast cancer offered by this type of surgery becomes more important. With respect to its prophylactic nature, and since the first report by Lund et al. [4] 28 years ago, there have been many reports studying the effect of breast reduction on the relative risk of developing breast cancer in the operated patient cohort. Despite the lack of randomized controlled trials and the absence of definitive proof, multiple observational studies do provide solid evidence that a critical reduction of breast and fatty tissue significantly lowers the breast cancer incidence in selected group of patients undergoing breast reduction surgery [4]-[11]. However, none of these reports have studied the impact of a specific surgical technique on the degree of risk reduction.

As numerous statistics and clinical studies have shown, the upper outer quadrant (UOQ) of the breast is the most frequent site of carcinoma encompassing about 45 percent of all breast cancer occurrences [12] [13] [14] [15]. When comparing data with respect to the year of publication, the incidence of UOQ breast cancer also seems to be on the rise. Starting with $31 \%$ in the early reports reaching $61 \%$ in the latest reports [16] [17].

Why does the highest percentage of breast cancers occur in the outer upper quadrant is still not fully understood. Some have found a higher level of genomic instability in this area promoting cancerization [18]. Other studies have attempted to identify environmental factors as a probable cause such as deodorants and other carcinogenic products applied to the axilla and affecting the outer breast quadrants [19], still no definitive explanation exists. Knowing that the incidence of breast cancer varies between the five breast quadrants where the majority of tumors occur in the lateral quadrants of the breast, and knowing that each breast reduction technique has its own pattern of gland excision, it would seem logical that not all will be equally effective in decreasing the risk of subsequent breast cancer.

In addition to providing reliable aesthetic and functional enhancement [20] [21] [22] [23] [24], one of the hallmarks of a Moufarrege total posterior pedicle breast reduction is the ability for a complete upper outer quadrant gland excision with inclusion of the most lateral axillary extension of the breast. This is accomplished through the development of thin mastectomy flaps that extend medially and laterally down to the muscle fascia. Most standard techniques will use thicker subcutaneous flaps especially at the base of the flap to optimise flap 
vascularisation which will act as a barrier to reach the tail of spence and also leave part of the gland attached to the subcutaneous tissue at this level.

The aim of this study is to assess the effects of the Moufarrege total posterior pedicle reduction mammaplasty on the post-operative incidence of breast cancer.

\section{Methods}

\subsection{Study Design and Sampling}

This study was performed with informed consent from the patients and institutional review board approval, and it was conducted in accordance with the Declaration of Helsinki. All data were treated in a confidential manner and the anonymity of the respondents was maintained.

We performed a retrospective study with primary and secondary care data collected between January 1, 1981, and December 3, 1997. Data were pooled from the medical records of Hotel-Dieu Hospital Montreal, Quebec, Canada and private clinic files.

\subsection{Eligibility Criteria}

Eligible patients included those that underwent a breast reduction surgery from the year 1981 to 1997 using the Moufarrege total posterior pedicle technique. All patients had a non-oncological indication for undergoing the procedure and had more than or equal to 250 grams of breast tissue resected as per the Quebec government requirements for a breast reduction.

\subsection{Measures}

We systematically reviewed all medical records, and with the collaboration of the health insurance of Quebec (RAMQ), were able to identify the most recent residential addresses of the eligible patients. We sent out a standardized questionnaire in two waves in 2008 to all patients. The questionnaire targeted information regarding the patient's age, the date and result of their last mammogram, any postoperative diagnosis of breast cancer (yes, no) and if yes date of diagnosis (continuous).Patients with missing medical records, incomplete chart data that was relevant to the outcome of the study and patients with a previous history of breast cancer were excluded from the study before sending out the questionnaire.

\subsection{Statistical Analysis}

We reviewed and edited the survey questionnaires prior to data entry, and we conducted reliability and consistency checks. A hospital chart review was performed to supplement all necessary information such as date of surgery, amount of breast tissue excised and perioperative medical course. The chart review was comprehensive of both hospital and private office notes. Prevalence estimates, expressed as numbers, percentages and means were estimated for all variables. Person-years (PY) of observation were calculated starting from the date of breast 
reduction surgery and ending at the date of death, or January 2008, whichever occurred first. Expected numbers of cancers were calculated by multiplying the age and calendar year specific PYs of observation times the corresponding cancer incidence rates of the general population [25]. Standardized incidence ratios (SIRs) were calculated as the ratio of observed-to-expected values and confidence intervals (CIs) calculated assuming a Poisson distribution. All statistical analyses were performed using the Statistical Software Package for Social Sciences (SPSS version 20).

\section{Operative Technique}

\subsection{Preoperative Consultation}

A pre-operative complete history and physical exam were performed for all patients. The surgeon met with every patient to discuss the operative procedure details, possible postoperative complications, goals and expectations, and a full consent was obtained.

Preoperative blood analysis, including complete blood count and coagulation profile was performed when appropriate. A mammography was done for all patients above 35 years of age and only those with normal mammograms underwent the operation. Patients with suspicious mammographic findings were seen in consultation and when needed referred to surgical oncology.

\subsection{Preoperative Markings}

The breasts were marked in the preoperative holding area. The new nipple position is placed at the level of the inframammary fold and a keyhole is drawn with the vertex marked $2 \mathrm{~cm}$ above the new nipple position. The curved part of the keyhole consists of an oval rather than a circle to provide a final circular shape after redraping and closure. The arms of the keyhole are drawn according to the degree of ptosis. There exists three categories of classification of ptosis and hypertrophy in the Moufarrège total posterior pedicle [23]. Category I, no ptosis, nipple at the level of the inframammary fold, keyhole angle $90^{\circ}-100^{\circ}$; category II, nipple below inframammary fold with the presence of breast volume at the level of the inframammary fold, keyhole angle $140^{\circ}-150^{\circ}$; category III, severe ptosis with breast emptiness at the level of the inframammary fold, keyhole angle $180^{\circ}$. The keyhole arms travel outward on the surface of the breast for a distance of $5 \mathrm{~cm}$ after which they curve inward in the manner of two meridians on the globe and meet at the inframammary fold (Figure 1). The area between the two arms is the amount of skin that needs to be resected (Figure 2).

\subsection{Operative Details}

All breast reductions were performed under general anesthesia by the same operator (R.M).

After prepping and draping the subcutaneous plane was infiltrated with $20 \mathrm{cc}$ epinephrine lidocaine $1 \%$ solution diluted in $20 \mathrm{cc}$ of normal saline. This facilitates our subcutaneous flap dissection which is performed in a relatively avascu- 
lar plane. After dis-epidermization of a $6 \mathrm{~cm}$ inferior dermal pedicle, a full thickness incision is made around that pedicle and along the drawings of the modified keyhole. Using a number 10 blade in a cleavage plane between the breast tissue and subcutaneous fat the internal, superior and external subcutaneous flaps are then developed to the most lateral, medial and upper borders of the breast till the muscle fascia. This will result in complete denudation of the breast with complete visualization of all quadrants providing maximum exposure for an easy and well calculated resection (Figure 3).

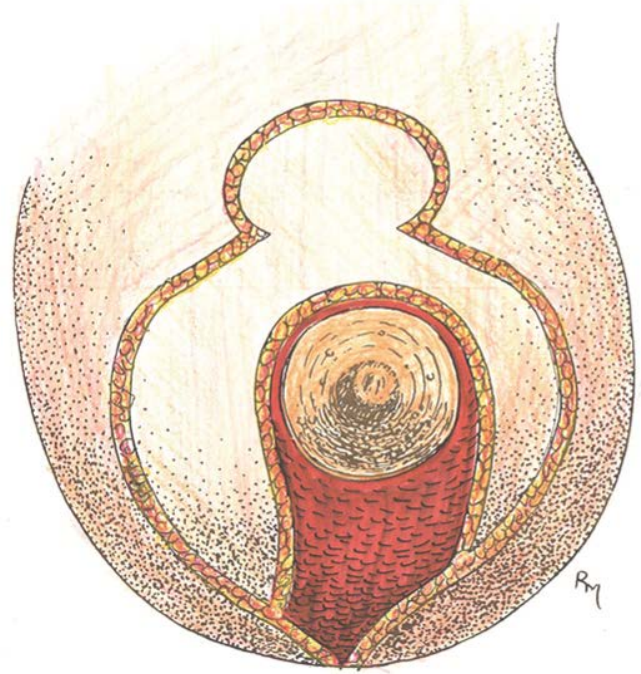

Figure 1. The keyhole arms curve inward in the manner of two meridians on the globe and meet at the inframammary fold. De-epithelialization of the central dermal pedicle is also shown.

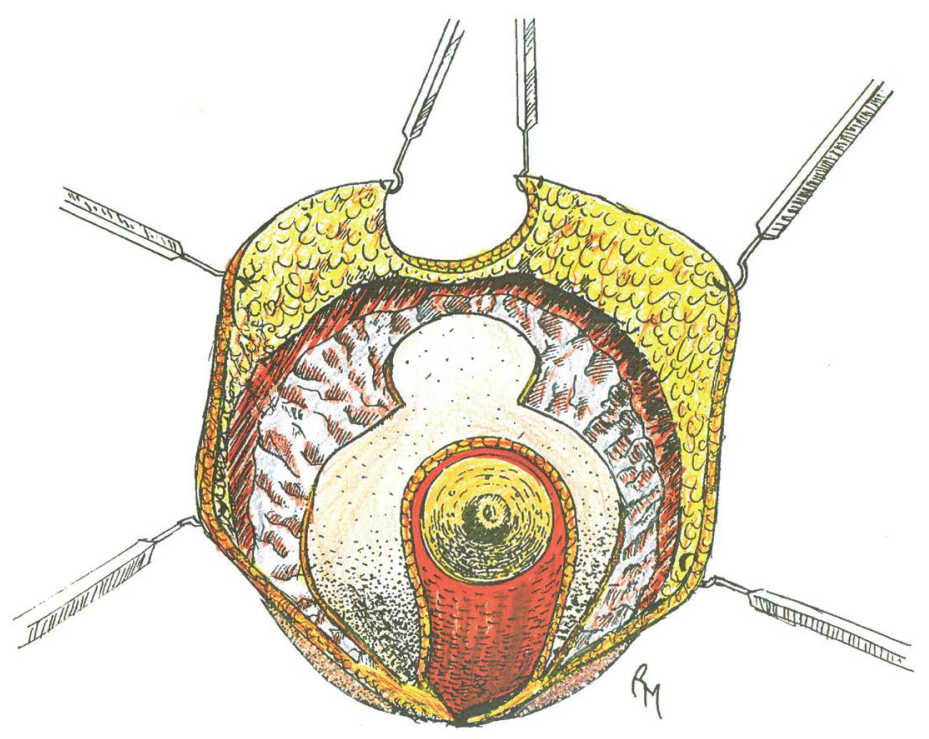

Figure 2. Development of the mastectomy flaps. The skin between the keyhole arms is resected. 


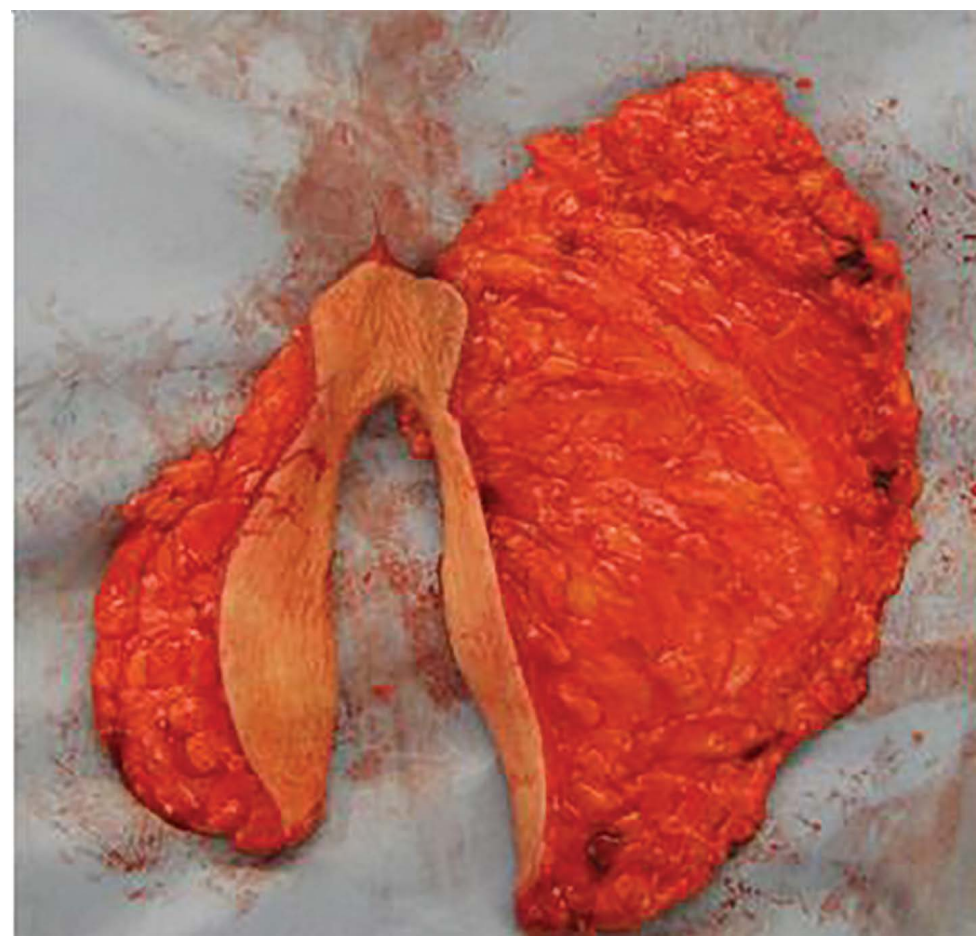

Figure 3. Left breast excised specimen with complete excision of the superolateral quadrant shown.

Medial resection is always minimal while lateral resection is done in total, following the extension of the breast tissue till the subaxillary region. Care is taken to preserve the areolar tissue above the lateral muscles at this level to ensure preservation of the intercostal nerves responsible for nipple erogenous sensation. No resection is performed in the inferior or superior quadrants. These two quadrants are the protected zones of the total posterior dermoglandular pedicle. After resection, breast reconstitution begins by suturing the NAC to its new position and the lateral and medial skin flaps are brought together in a vertical suture line. The vertical incision is transformed to an "inverted-T" whenever the vertical line exceeded $6 \mathrm{~cm}$ in length.

In conclusion what describes the Moufarrege Posterior Pedicle the most is the fact that the pedicle constitutes the totality of the remaining gland (Figure 4).

\section{Results}

The total number of patients meeting the inclusion criteria was 924 patients. The response rate was $60.38 \%(\mathrm{n}=558)$ and showed homogeneity through different age groups (Figure 5).

Mean patient age at the time of surgery was 32.8 years (14 to 82). Mean follow up was 17.7 years (11 to 27). Mean resection weight was 620 grs per breast (250 $1600 \mathrm{gr}$ ). No patient suffered from mastectomy flap necrosis. In some patients minor dehiscence at the inverted $\mathrm{T}$ junction were left to heal by secondary intention and required no further surgical intervention. Of the patients aged 50 years and more $93.33 \%$ had done a mammography in the two years prior to our sur- 


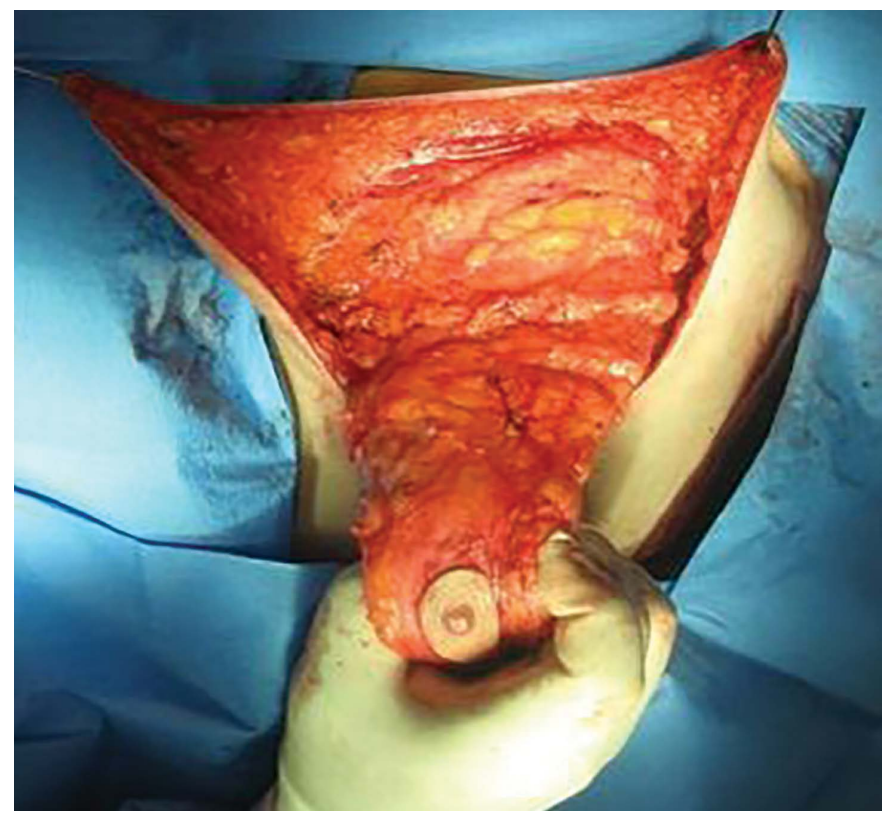

Figure 4. The remaining pedicle constitutes the totality of the breast.

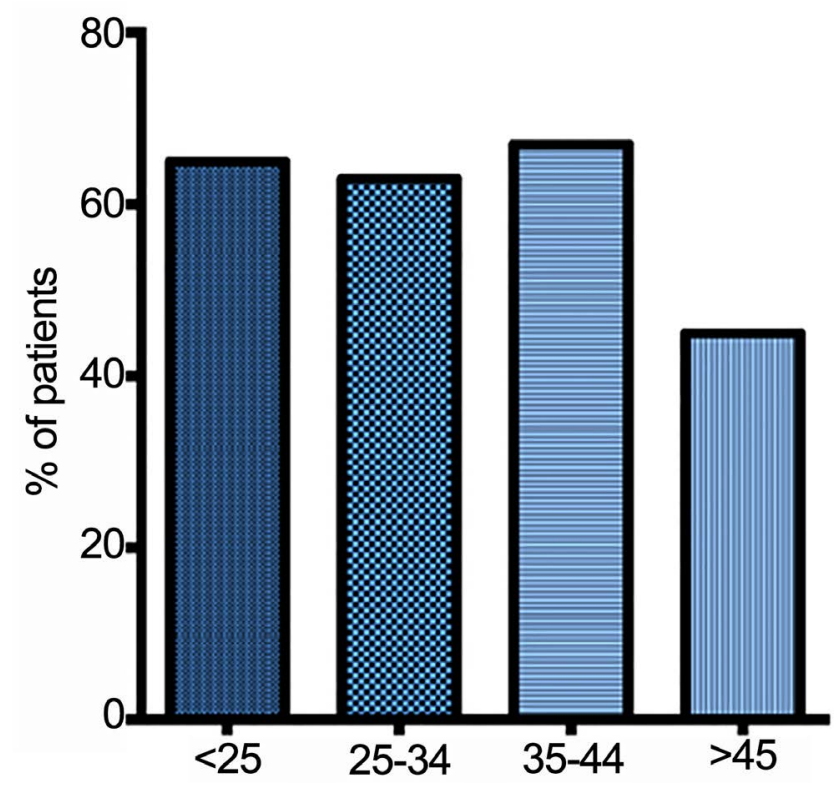

Figure 5. Patient response rate distribution by age at time of survey.

vey. Of the 558 patients who responded to the questionnaire, eight patients were diagnosed with having breast cancer in the postoperative follow up period. Standardized incidence ratio for breast cancer was 0.50 (CI: 0.21 - 0.98) showing a significant fifty percent reduction in the risk of breast cancer. Risk of subsequent breast cancer was inversely related to age at time of surgery (Table 1). Women aged 35 years or more at time of surgery had a greater and more statistically significant decrease in the risk for developing breast cancer ( $\operatorname{SIR}=0.36$ 95\% CI: 0.09 - 0.93) when compared to women aged less than 35 years of age at time of surgery (SIR $=0.78 \mathrm{CI}: 0.21-2.00$ ). The highest reduction in risk was 
seen in women aged 45 years or more at the time of surgery (SIR $=0.2$ CI: 0.00 1.13) while women aged less than 25 years at time of surgery showed no risk reduction (SIR = $1.08 \mathrm{CI}: 0.13$ - 3.9). The risk of breast cancer was also evaluated by age at last follow-up following breast reduction (Table 2). A significant risk reduction was mostly noted in patients aged 65 years or more at time of their last follow up (SIR = 0.27 CI: 0.03 - 0.98) while in patients less than 45 years of age at last follow up no significant reduction in risk was observed (SIR $=1.01 \mathrm{CI}$ : 0.02 $5.62)$.

\section{Discussion}

Many studies have attempted to prove a direct correlation between breast size and the risk of developing breast cancer [26] [27] [28]. Most have been inconclusive in identifying a definitive positive correlation. This may be due to the many different contributing risk factors, being hereditary or environmental, but is also due to the heterogeneous structure of the breast itself and the ratio of glandular mass to fatty tissue. It is known that women with mammographically dense breasts are at higher risk for cancer development [29] [30] [31] those women might have moderate size breasts and do not necessarily present with breast hypertrophy. On the other hand the fat content of the breast might also further contribute to the development of cancer by the conversion of androgens

Table 1. Standardized incidence ratios and 95\% confidence intervals for Breast cancer by Age at time of surgery.

\begin{tabular}{cccccc}
\hline Age at time & Number & \multicolumn{2}{c}{ Breast Cancer } & \\
\hline of surgery & of patients & Observed & Expected & SIR & $95 \%$ CI \\
\hline$<25$ & 205 & 2 & 1.85 & 1.08 & $0.13-3.90$ \\
$25-34$ & 133 & 2 & 3.25 & 0.61 & $0.07-2.22$ \\
$35-44$ & 140 & 3 & 5.99 & 0.50 & $0.10-1.46$ \\
$\geq 45$ & 80 & 1 & 4.93 & 0.20 & $0.00-1.13$ \\
Total & 558 & 8 & 16.02 & 0.5 & $0.21-0.98$ \\
\hline
\end{tabular}

SIR: Standardized incidence ratio, CI: Confidence Interval.

Table 2. Standardized incidence ratios and 95\% confidence intervals for breast cancer by Age at last follow-up.

\begin{tabular}{cccccc}
\hline Age at last & Number & \multicolumn{2}{c}{ Breast Cancer } \\
\hline follow-up & of patients & Observed & Expected & SIR & $95 \%$ CI \\
\hline$<45$ & 176 & 1 & 0.99 & 1.01 & $0.02-5.62$ \\
$45-54$ & 156 & 1 & 2.93 & 0.34 & $0.00-1.90$ \\
$55-64$ & 118 & 4 & 4.79 & 0.83 & $0.22-2.13$ \\
$\geq 65$ & 108 & 2 & 7.31 & 0.27 & $0.03-0.98$ \\
Total & 558 & 8 & 16.02 & 0.5 & $0.21-0.98$ \\
\hline
\end{tabular}

SIR: Standardized incidence ratio, CI: Confidence Interval. 
into estrogen through the action of the enzyme aromatase [32] and serve as repositories for fat-soluble carcinogens [33]. And even though no direct cause effect relationship between breast size or cup size and breast cancer can be established, we have enough indirect correlation that points to the fact that decreasing breast size through reduction or total excision does reduce the risk of developing breast cancer. In its extreme form of gland reduction, prophylactic mastectomy which removes almost all breast tissue was found to reduce the risk of breast cancer in high risk population by $90 \%$ [34] [35]. Another indirect correlation between breast size and cancer risk has been provided by the many observational studies that have reported on the effect of breast reduction surgery and breast cancer risk. In their series Lund et al. showed a $41 \%$ reduction in the incidence of breast cancer in a cohort of 1245 Danish women following breast reduction surgery [4]. Baasch et al., in an extension of Lund's series with 47 years of follow up showed a $39 \%$ risk reduction with the greatest reduction seen in patients with $>600$ gr removed [5]. Boice et al. studied the incidence of breast cancer among 31,910 breast reduction patients over a 28 year period compared to expected matched incidence SIR $=0.72$, (95\% CI 0.61 - 0.84), risk reduction was more pronounced in older women [6]. With a further follow up of Boice extended to 16 years, Fryzek et al. reported an SIR $=0.71$ (95\% CI $0.65-0.78)$ or a $29 \%$ reduction in relative risk [7]. In 2001 Brinton et al. performed an analysis of volume of tissue resection and risk of cancer with 161 cases compared to 483 controls. They found a direct correlation between the amount of breast tissue removed and the degree of risk reduction, were resections above $1600 \mathrm{~g}$ had a significantly lower risk of cancer than subjects with resections under $800 \mathrm{~g}$ (OR $=0.24,95 \%$ CI $0.1-0.5)$ [8]. The exact mechanism by which breast reduction tends to decrease the incidence of breast cancer is still not fully understood. One explanation could be decreasing the load of epithelial cells that have the potential to become cancerous [11] as well as the glandular breast units from which the cancer arises.

Our results are consistent with these previous ones, showing an overall 50\% decrease in the risk of breast cancer following breast reduction surgery. Furthermore the degree of risk reduction was proportional to age at time of surgery (Table 1). Women aged less than 25 years at time of surgery showed no decrease in risk (SIR $=1.08)$, while women older than 35 years at time of surgery showed a significant 64 percent decrease in subsequent breast cancer risk. An explanation for why younger women tend to benefit less with respect to risk reduction isn't very obvious. The increased breast cancer risk due to genetic predisposition or other factors associated with early-onset breast cancer conceivably may mask the protective effects of glandular tissue mass removal in this group of young patients. Also, since all of our patients who are older than 35 years of age at time of surgery had to undergo a pre-operative mammography, selection bias might have played a role in decreasing the risk in this group of women. Another reason might be different risk factors for breast hypertrophy and breast cancer among younger vs. older women. 
While women aged 65 years or more at last follow up had a significant 73 percent reduction in risk SIR $=0.27$ (CI: $0.03-0.98$ ), women less than 45 years of age at last follow up did not show any reduction in risk. This might possibly indicate that the reduction in risk comes later in life as the risk for developing breast cancer in older patients increases.

Besides the retrospective nature of this study and the possible selection bias of pre-op mammography and physical exam, other limitations would include the absence of data for confounding factors and general risk factors for developing breast cancer in our patients. This includes reproductive history, menarche, and family history. Also, the low number of breast cancer events in our series $(\mathrm{N}=8)$ affects the width of our confidence intervals.

We believe that having a patient cohort from 1981 to 1997 is one of the strength of our study which allowed us enough follow up time, mean 17.7 years, to be able to assess for the lifetime risk of breast cancer. Another strong point would be the standardized surgical technique, where all the patients were operated on by the same technique and the same surgeon.

\section{Conclusion}

Using the Moufarrege Total posterior pedicle we observed a significant reduction in cancer risk for all patients who responded to our survey and were aged 35 years or more at the time of surgery. In our opinion for a breast reduction surgery to be able to play a better prophylactic role in women with increased risk for breast cancer, the upper outer quadrant with its axillary extension should be fully excised. We also believe that breast reduction surgery should be encouraged in selected patients not only to improve fitness and wellbeing but also as an oncological preventive measure. In conclusion we recommend more trials in this area to better understand and assess the prophylactic role that breast reduction surgery can have on women with moderate to high risk for developing breast cancer who are unwilling to undergo total mastectomy.

\section{References}

[1] Chadbourne, E.B., Zhang, S., Gordon, M.J., et al. (2001) Clinical Outcomes in Reduction Mammaplasty: A Systematic Review and Meta-Analysis of Published Studies. Mayo Clinic Proceedings, 76, 503-510. https://doi.org/10.4065/76.5.503

[2] Bruhlmann, Y. and Tschopp, H. (1998) Breast Reduction Improves Symptoms of Macromastia and Has a Long-Lasting Effect. Annals of Plastic Surgery, 41, 240-245. https://doi.org/10.1097/00000637-199809000-00003

[3] Chao, J.D., Memmel, H.C., Redding, J.F., Egan, L., Odom, L.C. and Casas, L.A. (2002) Reduction Mamma Plasty Is a Functional Operation, Improving Quality of Life in Symptomatic Women: A Prospective, Single-Center Breast Reduction Outcome Study. Plastic and Reconstructive Surgery, 110, 1644-1652.

[4] Lund, K., Ewertz, M. and Schou, G. (1988) Breast Cancer Incidence Subsequent to Surgical Reduction of the Female Breast. Plastic and Reconstructive Surgery, 82, 564. https://doi.org/10.1097/00006534-198809000-00072

[5] Baasch, M., Nielsen, S.F., Engholm, G. and Lund, K. (1996) Breast Cancer Incidence 
Subsequent to Surgical Reduction of the Female Breast. British Journal of Cancer, 73, 961-963. https://doi.org/10.1038/bjc.1996.172

[6] Boice, J.D., Persson, I., Brinton, L.A., et al. (2000) Breast Cancer Following Breast Reduction Surgery in Sweden. Plastic and Reconstructive Surgery, 106, 755-762. https://doi.org/10.1097/00006534-200009020-00001

[7] Fryzek, J.P., Weimin, Y., Nyren, O., et al. (2006) A Nationwide Study of Breast Cancer Incidence Following Breast Reduction Surgery in a Large Cohort of Swedish Women. Breast Cancer Research and Treatment, 97, 131-134. https://doi.org/10.1007/s10549-005-9099-2

[8] Brinton, L.A., Persson, I., Boice, J.J.D., McLaughlin, J.K. and Fraumeni, J.J.F. (2001) Breast Cancer Risk in Relation to Amount of Tissue Removed during Breast Reduction Operations in Sweden. Cancer, 91, 478-483. https://doi.org/10.1002/1097-0142(20010201)91:3<478::AID-CNCR1025>3.0.CO;2$\underline{5}$

[9] Brinton, L.A., Malone, K.E., Coates, R.J., et al. (1996) Breast Enlargement and Reduction: Resultsfrom a Breast Cancer Case-Control Study. Plastic and Reconstructive Surgery, 97, 269-275. https://doi.org/10.1097/00006534-199602000-00001

[10] Brown, M.H., Weinberg, M., Chong, N., Levine, R. and Holowaty, E. (1999) A Cohort Study of Breast Cancer Risk in Breast Reduction Patients. Plastic \& Reconstructive Surgery, 103, 1674-1681. https://doi.org/10.1097/00006534-199905000-00015

[11] Tarone, R.E., Lipworth, L., Young, V.L. and McLaughlin, J.K. (2004) Breast Reduction Surgery and Breast Cancer Risk: Does Reduction Mammaplasty Have a Role in Primary Prevention Strategies for Women at High Risk of Breast Cancer? Plastic and Reconstructive Surgery, 113, 2104-2110.

[12] Truscott, B.M. (1947) Carcinoma of the Breast. British Journal of Cancer, 1, 129.

[13] Harnett, W.L. (1948) A Statistical Report on 2529 Cases of Cancer of the Breast. British Journal of Cancer, 2, 212.

[14] Nohrman, B.A. (1949) Cancer of the Breast. Acta Radiologica, Suppl 77.

[15] Smithers, D.W., Rigby-Jones, P., Galton, D.A.G. and Payne, P.M. (1952) Cancer of the Breast. The British Journal of Radiology, Suppl 4.

[16] Darbre, P.D. (2005) Recorded Quadrant Incidence of Female Breast Cancer in Great Britain Suggests a Disproportionate Increase in the Upper Outer Quadrant of the Breast. Anticancer Research, 25, 2543-2550.

[17] Aljarrah, A. and Miller, W.R. (2014) Trends in the Distribution of Breast Cancer over Time in the Southeast of Scotland and Review of the Literature. E Cancer Medical Science, 8, 427.

[18] Ellsworth, D.L., Ellsworth, R.E., Love, B., et al. (2004) Outerbreast Quadrants Demonstrate in Creased Levels of Genomic Instability. Annals of Surgical Oncology, 11, 861-868.

[19] Darbre, P.D. and Charles, A.K. (2010) Environmental Oestrogens and Breast Cancer: Evidence for Combined Involvement of Dietary, Household and Cosmetic Xenoestrogens. Anticancer Research, 30, 815-827.

[20] Moufarrege, R. (1979) A New Reduction Mammaplasty with a Vertical Posterior Pedicle. Quebec Society of Plastic Surgery Convention, Quebec.

[21] Moufarrege, R., Muller, G.H., Beauregard, G., Bosse, J.P. and Papillon, J. (1982) Mammaplasty with a Lower Dermal-Glandular Pedicle. Annales De Chirurgie Plastique, 27, 249-254.

[22] Moufarrege, R., Beauregard, G., Bosse, J.P., et al. (1985) Reduction Mammaplasty by 
the Total Dermoglandular Pedicle. Aesthetic Plastic Surgery, 9, 227-232. https://doi.org/10.1007/BF01570855

[23] Moufarrege, R.J. (2003) Moufarrege Total Posterior Pedicle Breast Reduction. eMedicine. http://emedicine.medscape.com/article/1275321-overview

[24] Sinno, H., Botros, E. and Moufarrege, R. (2013) The Effects of Moufarrege Total Posterior Pedicle Reduction Mammaplasty on Breast Feeding: A Review of 931 Cases. Aesthetic Surgery Journal, 33, 1002-1007.

[25] Cancer Statistics Review 1975-2007. http://seer.cancer.gov/csr/1975_2007/results_merged/sect_04_breast.pdf

[26] Egan, K.M., Newcomb, P.A., Titus-Ernstoff, L., et al. (1999) The Relation of Breast Size to Breast Cancer Risk in Postmenopausal Women (United States). Cancer Causes \& Control, 10, 115-118. https://doi.org/10.1023/A:1008801131831

[27] Tavani, A., Pregnolato, A., La Vecchia, C., Negri, E., Favero, A. and Franceschi, S. (1996) Breast Size and Breast Cancer Risk. European Journal of Cancer Prevention, 5, 337-342. https://doi.org/10.1097/00008469-199610000-00005

[28] Trichopoulos, D. and Lipman, R.D. (1992) Mammary Gland Mass and Breast Cancer Risk. Epidemiology, 3, 523-526. https://doi.org/10.1097/00001648-199211000-00011

[29] Byrne, C., Schairer, C., Wolfe, J., et al. (1995) Mammographic Features and Breast Cancer Risk: Effects with Time, Age, and Menopausestatus. Journal of the National Cancer Institute, 87, 1622-1629. https://doi.org/10.1093/jnci/87.21.1622

[30] Thurfjell, E., Hsieh, C.C., Lipworth, L., Ekbom, A., Adami, H.O. and Trichopoulos, D. (1996) Breast Size and Mammographic Pattern in Relation to Breast Cancer Risk. European Journal of Cancer Prevention, 5, 37-41.

[31] Vachon, C.M., Kuni, C.C., Anderson, K., Anderson, V.E. and Sellers, T.A. (2000) Association of Mammographically Defined Percent Breast Density with Epidemiologic Risk Factors for Breast Cancer (United States). Cancer Causes \& Control, 11, 653-662. https://doi.org/10.1023/A:1008926607428

[32] Sasano, H. and Ozaki, M. (1997) Aromatase Expression and Its Localization in Human Breast Cancer. The Journal of Steroid Biochemistry and Molecular Biology, 61, 293-298. https://doi.org/10.1016/S0960-0760(97)80026-X

[33] Martin, F.L., Venitt, S., Carmichael, P.L., et al. (1997) DNA Damage in Breast Epithelial Cells: Detection by the Single-Cell Gel (Comet) Assay and Induction by Human Mammary Lipid Extracts. Carcinogenesis, 18, 2299-2305. https://doi.org/10.1093/carcin/18.12.2299

[34] Hartmann, L.C., Schaid, D.J., Woods, J.E., et al. (1999) Efficacy of Bilateral Prophylactic Mastectomy in Women with a Family History of Breast Cancer. The New England Journal of Medicine, 340, 77-84. https://doi.org/10.1056/NEJM199901143400201

[35] Hartmann, L.C., Sellers, T.A., Schaid, D.J., et al. (2001) Efficacy of Bilateral Prophylactic Mastectomy in BRCA1 and BRCA2 Gene Mutation Carriers. Journal of the National Cancer Institute, 93, 1633-1637. https://doi.org/10.1093/jnci/93.21.1633 